\title{
Introducing Global and Regional Mainstreaminess for Improving Personalized Music Recommendation
}

\author{
Markus Schedl \\ Johannes Kepler University \\ Linz, Austria \\ markus.schedl@jku.at
}

\author{
Christine Bauer \\ Johannes Kepler University \\ Linz, Austria \\ christine.bauer@jku.at
}

\begin{abstract}
The music mainstreaminess of a user reflects how strong a user's listening preferences correspond to those of the larger population. Considering that music mainstream may be defined from different perspectives and on various levels, e.g., geographical (charts of a country), genre ("Indie charts"), or distribution channel (radio charts vs. download charts), we study how the user's music mainstreaminess influences the quality of music recommendations.

The paper's contribution is three-fold. First, we propose 11 novel mainstreaminess measures characterizing music listeners, considering both a global and a country-specific basis. To this end, we model preference profiles (as a vector over artists) for users, countries, and globally, incorporating artist frequency, listener frequency, and a newly proposed TF-IDF-inspired weighting function, which we call artist frequency-inverse listener frequency (AF-ILF). The resulting preference profile for each user $u$ is then related to the respective country-specific and global preference profile using fractionbased approaches, symmetrized Kullback-Leibler divergence, and Kendall's $\tau$ rank correlation, in order to quantify $u$ 's mainstreaminess. Second, we demonstrate country-specific peculiarities of these mainstreaminess definitions. Third, we show that incorporating the proposed global and country-specific mainstreaminess measures into the music recommendation process can notably improve accuracy of rating prediction.
\end{abstract}

\section{CCS CONCEPTS}

- Information systems $\rightarrow$ Multimedia information systems; Personalization; Recommender systems; Personalization; Rank aggregation; Clustering and classification; Collaborative filtering; Social recommendation; $\bullet$ Social and professional topics $\rightarrow \mathbf{C u l}-$ tural characteristics; $\bullet$ Applied computing $\rightarrow$ Sound and music computing;

\section{KEYWORDS}

Music mainstreaminess, music recommender systems, artist frequencyinverse listener frequency, popularity

\section{ACM Reference format:}

Markus Schedl and Christine Bauer. 2017. Introducing Global and Regional Mainstreaminess for Improving Personalized Music Recommendation. In

Permission to make digital or hard copies of part or all of this work for personal or classroom use is granted without fee provided that copies are not made or distributed for profit or commercial advantage and that copies bear this notice and the full citation on the first page. Copyrights for third-party components of this work must be honored.

For all other uses, contact the owner/author(s).

MoMM '17, December 4-6, 2017, Salzburg, Austria

(C) 2017 Copyright held by the owner/author(s)

ACM ISBN 978-1-4503-5300-7/17/12.

https://doi.org/10.1145/3151848.3151849
Proceedings of The 15th International Conference on Advances in Mobile Computing \& Multimedia, Salzburg, Austria, December 4-6, 2017 (MoMM '17), 8 pages.

https://doi.org/10.1145/3151848.3151849

\section{INTRODUCTION}

In the era of digitalization, music has become easier to access than ever: a tremendous number of musical recordings are readily available to consume on online music platforms such as YouTube, Spotify, or iTunes. This opportunity to access a large number of musical works, though, results in information overload and users require novel mechanisms and strategies to choose from the "deep blue sea of musical works" [30]. Music recommender systems (MRS) have, thus, become a significant topic both in research as well as in industry, over the past few years $[5,8,33]$.

In general, recommender systems are meant to assist users in searching, sorting, and filtering the vast amount of information available [22]. MRS are specifically built to assist users in navigating through the myriad of available musical recordings and provide them with suggestions (and/or automatically generated playlists) that would fit the respective user's interest [33]. Thereby, "[t]he success of a music recommender system (RS) depends on its ability to propose the right music, to the right user, at the right moment" [19].

Various automatic approaches to music recommendation have been proposed [35]. Thereby, most MRS rely mainly on collaborative filtering [20] or on information about music items (i.e., content-based filtering [4]) [35]. For instance, content-based MRS may consider acoustic similarity information on the song level [38], or genre or artist similarity [21]. MRS employing collaborative filtering do not require exogenous information about neither users nor music items. Instead, a user is suggested music listened to by users with similar preferences and/or listening patterns [25].

An approach that is particularly applicable in hit-driven domains such as the music industry is popularity-based recommendation. This approach assumes that a random user is more likely to like a very popular music item than one of the far less popular items $[8,34]$. Popularity-based MRS approaches are widely adopted to complement other approaches in cold start situations, when there is limited information about new users and/or items available in the system $[10,39]$. One approach for considering popularity in the music domain is to describe music listeners "in terms of the degree to which they prefer music items that are currently popular or rather ignore such trends" [29]. Harnessing music mainstreaminess in combination with collaborative filtering techniques tends to deliver better results with respect to music recommendation accuracy and rating prediction error than pure collaborative filtering approaches alone $[13,32,34,37]$. 
However, a limitation of existing work on quantifying a user's music mainstreaminess is that music mainstream is viewed from a global perspective. There exist regional peculiarities to mainstream, though, as music consumption behavior is affected by culturally influenced music preferences, market regulations, local radio airplay, etc. (e.g., [7, 16, 26, 36]). With respect to the music recommendation research domain, the definition of specific measures that can capture a user's mainstreaminess (i) on both, a global and a countryspecific level, and (ii) in ways that can easily be operationalized in music recommendation is a new target of research.

Calling on this, the main contributions of this paper are threefold: (i) the definition of several novel measures for user mainstreaminess, considering both a global and a regional, countryspecific basis, (ii) illustrating country-specific peculiarities of these mainstreaminess definitions, and (iii) analyzing the performance of the proposed mainstreaminess measures for personalized music recommendation.

The remainder of the paper is organized as follows. In Section 2, we provide a brief overview over existing work on mainstreaminess and popularity in music recommendation. We then detail our proposed mainstreaminess measures in Section 3 and provide examples that show their value to distill the regional mainstream, in addition to a global one. Section 4 shows how to exploit the proposed mainstreaminess measures in collaborative filtering recommendation and highlights the additional values of doing so. Eventually, we round off the paper in Section 5 with a conclusion and directions for future research.

\section{CONCEPTUAL FOUNDATIONS AND RELATED WORK}

\subsection{Music Popularity and Mainstreaminess}

In the context of recommender systems, popularity-based approaches are widely adopted in numerous domains, including music [10, 18, 39], news [40], or product recommendation in e-commerce in general [1]. Popularity is thereby typically constructed as a general consensus of a group's attitude about entities [18].

While various ways exist to define and measure popularity (for instance, in terms of sales figures, media coverage, etc.), in the field of MRS, music popularity is frequently characterized by using the total playcounts of a music item, cf. [8].

With respect to music popularity by using playcounts, the long tail concept as described in [2] is specifically applicable to the (online) music industry [9]; on online music platforms there is a concentration of playcounts on the most popular music items (the head) and then there is a long tail of less popular items [6, 8].

A more general concept to popularity concentration is referred to as mainstream. Although literature in the field of popular music studies and popular music cultures references to mainstream frequently, the term itself remains rather poorly defined, cf. e.g., [3] According to the Oxford Dictionaries, mainstream is defined as "The ideas, attitudes, or activities that are shared by most people and regarded as normal or conventional". Due to the strong connection of the concepts, the terms mainstream and long tail are often interchangeably used. The mainstream is thereby frequently also referred to with other terms and phrases (e.g., hits [8], the head [12]) to circumscribe the phenomenon; the overall concept is also called, for instance, the hit-driven paradigm [8], the long-tail concept $[2,8]$, etc.

In MRS research, the user feature music mainstreaminess of a user $[13,34]$ essentially describes whether and how strong a user's music listening preferences correspond to those of the overall population. While other listening-centric features, for instance, serendipity [41] or novelty [11], are frequently exploited when modeling a user's music consumption behavior and providing music recommendations, music mainstreaminess is a rather new target of research [13, 34, 37]. Thereby, the mainstreaminess feature is used to analyze a user's ranking of music items and compare it with the overall ranking of artists, albums, or tracks [37].

\subsection{Related Work on the Quantification of Music Mainstreaminess}

Formal definitions to measure the level of music mainstreaminess of a user are scarce in literature (e.g., [32, 34, 37]). Most existing approaches quantify music mainstreaminess as fractions of the target user's playcounts among the playcounts of the overall population. A limitation of this approach is that it disproportionately privileges the absolute top hits [32], which is problematic in longtail economies [2] such as the music economy, where there is a high concentration of demands on the most popular items and a long tail of less popular items. Privileging the top hits leads to lower performance when considering fraction-based user models of mainstreaminess in collaborative filtering approaches [32].

To overcome this limitation, Schedl and Bauer [32] proposed measurement approaches based on rank-order correlation and KullbackLeibler $(K L)$ divergence. However, also their work shares with existing fraction-based approaches to quantify mainstreaminess that music mainstream is viewed from a global perspective and does not take regional peculiarities of music mainstream into account.

\subsection{Music Mainstreaminess and Cultural Aspects}

As human preferences and behavior are rooted and embodied in culture [17], also music preferences and music consumption behavior are affected by cultural aspects $[14,16,36]$. Not only cultural aspects, but also other regional (e.g., country-specific) mechanisms that affect music consumption (e.g., market regulations, access to music items, radio airplay), shape the regional mainstream.

Against this background, we focus on country-specific differences in the paper at hand.

Closest to our work is the study presented in [37], which analyzes the recommendation performance of mainstreaminess (spelled "mainstreamness") and a user's country, among other features. Our work significantly differs from [37] in various regards: First, we use an open dataset to allow for replication. Second, [37] propose only one global mainstreaminess measure that compares a user's preferences to the overall dataset (global population), while we define mainstreaminess in various ways (based on fractional, divergence, and rank correlation functions) and at various levels (global and country-specific). Third, we also propose a novel weighting approach based on "inverse listening frequency" that highlights artists popular in a specific country, thus, contributing to its mainstream, but not necessarily on a global level. 


\begin{tabular}{|lr|lr|}
\hline Artist & AF & Artist & LF \\
\hline The Beatles & $2,985,509$ & Radiohead & 24,829 \\
Radiohead & $2,579,453$ & Nirvana & 24,249 \\
Pink Floyd & $2,351,436$ & Coldplay & 23,714 \\
Metallica & $1,970,569$ & Daft Punk & 23,661 \\
Muse & $1,896,941$ & Red Hot Chili Peppers & 22,609 \\
Arctic Monkeys & $1,803,975$ & Muse & 22,429 \\
Daft Punk & $1,787,739$ & Queen & 21,778 \\
Coldplay & $1,755,333$ & The Beatles & 21,738 \\
Linkin Park & $1,691,122$ & Pink Floyd & 21,129 \\
Red Hot Chili Peppers & $1,627,851$ & David Bowie & 20,602 \\
\hline
\end{tabular}

Table 1: Global top artists in the LFM-1b dataset, according to artist frequency (AF) and listener frequency (LF), considering the 53,258 users with country information.

\begin{tabular}{|lr|}
\hline Artist & AF \\
\hline Stam1na & 105,633 \\
In Flames & 97,645 \\
CMX & 90,032 \\
Kotiteollisuus & 82,309 \\
Turmion Kätilöt & 78,722 \\
Amorphis & 78,159 \\
Nightwish & 75,742 \\
Mokoma & 73,453 \\
Muse & 69,507 \\
Metallica & 69,499 \\
\hline Artist & LF \\
\hline Metallica & 703 \\
Nightwish & 695 \\
Muse & 693 \\
Daft Punk & 675 \\
Queen & 671 \\
System of a Down & 663 \\
Coldplay & 634 \\
Nirvana & 614 \\
Pendulum & 613 \\
Iron Maiden & 609 \\
\hline Artist & AF-ILF \\
\hline St. Hood & 70.526 \\
The Sun Sawed in $1 / 2$ & 67.490 \\
tiko- $\mu$ & 66.546 \\
Worth the Pain & 66.058 \\
Cutdown & 65.247 \\
Katariina Hänninen & 64.955 \\
Game Music Finland & 64.835 \\
Daisuke Ishiwatari & 63.565 \\
Altis & 63.235 \\
Redrum-187 & 62.428 \\
\hline & \\
\hline
\end{tabular}

(a) Finland (1,407 users)

\begin{tabular}{|lr|}
\hline Artist & AF \\
\hline Radiohead & 68,160 \\
The Beatles & 65,498 \\
Pink Floyd & 60,558 \\
Fabrizio De André & 53,928 \\
Muse & 48,168 \\
Depeche Mode & 42,586 \\
Afterhours & 42,473 \\
Verdena & 42,338 \\
Sigur Rós & 41,748 \\
Arctic Monkeys & 39,755 \\
\hline Artist & LF \\
\hline Radiohead & 556 \\
Pink Floyd & 539 \\
The Beatles & 505 \\
David Bowie & 500 \\
Muse & 500 \\
Nirvana & 497 \\
Coldplay & 475 \\
The Cure & 466 \\
Depeche Mode & 459 \\
Daft Punk & 457 \\
\hline Artist & AF-ILF \\
\hline CaneSecco & 68.451 \\
DSA Commando & 66.049 \\
Veronica Marchi & 65.864 \\
Train To Roots & 65.459 \\
Alessandro Raina & 64.228 \\
Machete Empire & 63.915 \\
Danti & 62.958 \\
Dargen D'Amico & 62.453 \\
宝塚歌劇団 • 苖組 & 62.228 \\
Aquefrigide & 61.663 \\
\hline & \\
\hline
\end{tabular}

(b) Italy (972 users)

\begin{tabular}{|lr|}
\hline Artist & AF \\
\hline Pink Floyd & 68,887 \\
Metallica & 42,784 \\
Daft Punk & 42,020 \\
Iron Maiden & 34,174 \\
Radiohead & 31,390 \\
Massive Attack & 30,669 \\
The Beatles & 27,951 \\
Opeth & 25,744 \\
Depeche Mode & 25,075 \\
Dream Theater & 24,286 \\
\hline Artist & LF \\
\hline Pink Floyd & 292 \\
Radiohead & 289 \\
Metallica & 268 \\
Coldplay & 261 \\
Nirvana & 251 \\
Massive Attack & 249 \\
The Beatles & 240 \\
Red Hot Chili Peppers & 240 \\
Queen & 238 \\
Led Zeppelin & 236 \\
\hline Artist & AF-ILF \\
\hline Cüneyt Ergün & 64.473 \\
Floyd Red Crow Westerman & 61.955 \\
Frrat Tanış & 58.666 \\
Acil Servis & 58.439 \\
Taste (Rory Gallager) & 58.366 \\
Mezarkabul & 57.799 \\
Rachmaninoff Sergey & 57.733 \\
Mabel Matiz & 57.619 \\
Grup Yorum & 56.855 \\
Yüzyüzeyken Konuşuruz & 56.748 \\
\hline & \\
\hline
\end{tabular}

(c) Turkey (479 users)

Table 2: Top artists for selected countries, according to artist frequency (AF), listener frequency (LF), and artist frequencyinverse listener frequency (AF-ILF).

\section{FORMALIZING MAINSTREAMINESS}

When describing how well a user's listening preferences reflect those of an overall population, e.g., globally or within a country, what is considered mainstream depends on the selection of a population; this is a phenomenon which we will also show in our analysis. Consequently, we propose several quantitative measures for user mainstreaminess, both on a global and on a country-specific level, depending on the selection of the population against which the user is compared. Our approach is inspired by the well-established monotonicity assumptions in text processing and information retrieval [28] - the TF-IDF (term frequency-inverse document frequency) weighting. Based on this assumption, our proposed mainstreaminess measures rely on the concepts of artist frequency $(A F)$, listener frequency $(L F)$, and artist frequency-inverse listener frequency (AF-ILF).

We define $A F_{a, U}$ as the sum of the number of tracks by artist $a$ listened to by a set of users $U$. Note that $U$ may be a single user $u$, all users in a country $c$, or the entirety of users in the collection (i.e., the global population $g$ ). Accordingly, we define $L F_{a, U}$ as the number of 


\begin{tabular}{|c|c|}
\hline Abbr. & Formula \\
\hline$F_{g: A F, u: A F}$ & $1-\frac{1}{|A|} \cdot \sum_{a \in A} \frac{\left|\widehat{A F_{a, u}}-\widehat{A F_{a, g}}\right|}{\max \left(\widehat{A F_{a, u}}, \widehat{A F_{a, g}}\right)}$ \\
\hline$F_{g: A F, u: A F \cdot I L F}$ & $1-\frac{1}{|A|} \cdot \sum_{a \in A} \frac{\left|A{\widehat{F \cdot I L F_{a, u, g}}}-\widehat{A F}_{a, g}\right|}{\max \left(\widehat{A F \cdot I L F_{a, u}, g}, \widehat{A F}_{a, g}\right)}$ \\
\hline$F_{g: A F \cdot I L F, u: A F \cdot I L F}$ & $1-\frac{1}{|A|} \cdot \sum_{a \in A} \frac{\mid A \widehat{F \cdot I L F_{a, u, g}}-A \widehat{\max \left(\overline{A L F_{a, g}, g} \mid\right.}}{\left.A \widehat{F \cdot I L F_{a, u}}, \overline{A F \cdot I L F_{a, g}}\right)}$ \\
\hline$F_{c: A F, u: A F}$ & $1-\frac{1}{|A|} \cdot \sum_{a \in A} \frac{\left|\widehat{A F_{a, u}}-\widehat{A F_{a, c}}\right|}{\max \left(\widehat{A F_{a, u}}, \widehat{A F_{a, c}}\right)}$ \\
\hline$F_{c: A F \cdot I L F, u: A F \cdot I L F}$ & $1-\frac{1}{|A|} \cdot \sum_{a \in A} \frac{\left|A \widehat{F \cdot I L F_{a, u}}-A \widehat{A \cdot I L F_{a, c}, g}\right|}{\max \left(\widehat{A F \cdot I L F_{a, u}}, \overline{A F \cdot I L F_{a, c}, g}\right)}$ \\
\hline$D_{g: A F, u: A F}$ & $\frac{1}{2} \cdot\left(\sum_{a \in A} \widehat{A F_{a, u}} \cdot \log \frac{\widehat{A F_{a, u}}}{\widehat{A F_{a, g}}}+\sum_{a \in A} \widehat{A F_{a, g}} \cdot \log \frac{\widehat{A F_{a, g}}}{\overline{A F_{a, u}}}\right)^{-1}$ \\
\hline$D_{c: A F, u: A F}$ & $\frac{1}{2} \cdot\left(\sum_{a \in A} \widehat{A F_{a, u}} \cdot \log \frac{\widehat{A F_{a, u}}}{\widehat{A F_{a, c}}}+\sum_{a \in A} \widehat{A F_{a, c}} \cdot \log \frac{\widehat{A F_{a, c}}}{\widehat{A F_{a, u}}}\right)^{-1}$ \\
\hline$D_{c: A F \cdot I L F, u: A F \cdot I L F}$ & $\frac{1}{2} \cdot\left(\sum_{a \in A} A \widehat{A \cdot I L F_{a, u}}, g \cdot \log \frac{A \overline{F \cdot I L F_{a, u}}}{A \widehat{F \cdot I L F_{a, c}}}+\sum_{a \in A} A \widehat{A \cdot I L F_{a, c}, g} \cdot \log \frac{A \overline{F \cdot I L F_{a, c}, g}}{A \overline{F \cdot I L F_{a, u}}}\right)^{-1}$ \\
\hline$C_{g: A F, u: A F}$ & $\tau\left(\operatorname{ranks}\left(P P_{g}^{A F}\right), \operatorname{ranks}\left(P P_{u}^{A F}\right)\right)$ \\
\hline$C_{c: A F, u: A F}$ & $\tau\left(\operatorname{ranks}\left(P P_{c}^{A F}\right), \operatorname{ranks}\left(P P_{u}^{A F}\right)\right)$ \\
\hline$C_{c: A F \cdot I L F, u: A F \cdot I L F}$ & $\tau\left(\operatorname{ranks}\left(P P_{u, c}^{A F I L F}\right), \operatorname{ranks}\left(P P_{c, g}^{A F \cdot I L F}\right)\right)$ \\
\hline
\end{tabular}

Table 3: Proposed music mainstreaminess measures on the user level. Terms denote the following: $F$ stands for the fractionbased approach, $D$ refers to the symmetrized Kullback-Leibler divergence approach, and $C$ is used as abbreviation for the approaches based on rank-order correlation according to Kendall's $\tau$. $A$ is a list of all artists; $\widehat{A F}$ denotes the sum-to-unity normalized $A F$ value; $\operatorname{ranks}\left(P P_{u}^{W}\right)$ represents the real-valued preference profile converted to ranks, i.e. the vector containing all normalized item frequencies of user $u$, with respect to the frequency weighting approach $W(A F$ or $L F)$; in case of $A F \cdot I L F$,

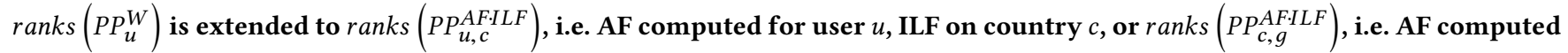
on country $c$, ILF globally. Note that we invert the values of some measures $(F$ and $D)$ in order to ensure that higher values always indicate closer to the mainstream.

listeners of artist $a$ within a user population $U$. And we eventually define $A F \cdot I L F_{a, U_{1}, U_{2}}$ as in Equation 1 . We set $A F \cdot I L F_{a, U_{1}, U_{2}}=0$ iff $L F_{a, U_{2}}=0$.

$$
A F \cdot I L F_{a, U_{1}, U_{2}}=\log \left(1+A F_{a, U_{1}}\right) \cdot \log \left(1+\frac{\left|U_{2}\right|}{L F_{a, U_{2}}}\right)
$$

Note that $U_{1}$ and $U_{2}$ may represent a single user, all users in the same country, or all users in the dataset. Therefore, this definition allows us to easily formalize both the global and the regional definitions of mainstreaminess, by varying $U_{1}$ and $U_{2}$. The $I L F$ weighting term can be integrated when computing the preference profile for a user or for a country, e.g., $A F \cdot I L F_{a, u, c}$, where $U_{1}$ contains only the user $u$ and $U_{2}$ all users in country $c$ (to which $u$ belongs), or $A F \cdot I L F_{a, c, g}$, where $U_{1}$ is composed of all users in country $c$ (to which $u$ belongs) and $U_{2}$ of all users in the dataset. Using ILF is motivated by the fact that, when determined by $A F_{a, c}$ or $L F_{a, c}$, the top artists in each country $c$ are often identical or very similar to the global top artists (cf. Tables 1 and 2). In order to uncover the respective country-specific mainstream, we therefore use $I L F_{a, g}$ to penalize globally popular artists.

Table 2 illustrates the effect of this weighting. It shows the top artists for Finland, Italy, and Turkey, in terms of $A F_{a, c}, L F_{a, c}$, and 
$A F \cdot I L F_{a, c, g}$, i.e., AF computed on the country level, ILF on the global level. Please note that artist IDs (on the $\mathrm{x}$-axis) are sorted with respect to their global popularity in regards to the respective measure (AF, LF, or AF-ILF). As can be seen, the AF and even more the LF measures are not suited well to distill the essential mainstream of a country, except maybe for countries such as Finland that show a very specific music taste far away from the global taste [31]. In contrast, AF-ILF is capable of identifying those artists that are popular in a specific country, but not worldwide.

Based on the above definitions, we compute preference profiles globally $\left(P P_{g}\right)$, for a country $\left(P P_{c}\right)$, and for a user $\left(P P_{u}\right)$. Given the LFM-1b dataset [30], these profiles are 585,095-dimensional vectors containing the AF, LF, or AF-ILF scores over all artists in the dataset. Figure 1 provides an example by visualizing the preference profiles including the top 50,000 artists for Finland, a country that does particularly not correspond to the global music mainstream. The black lines in the plots indicate the global AF, LF, or AF-ILF scores. As can be seen, the distributions of the AF- and LF-based preference profiles largely follow a trend similar to the global one. However, a second curve is indicative of a country-specific mainstream, in this case for Finland. In contrast, the AL-ILF weighting considerably increases the importance of globally less popular, but country-wise more popular artists (also see Table 2).

Exploiting the profiles, we propose three categories of mainstreaminess measures on the user level: fraction-based $(F)$, symmetrized Kullback-Leibler divergence $(D)$, and rank-order correlation according to Kendall's $\tau(C)$. The adoption of fraction-based measures is motivated by their easy interpretability (due to the share of overlap between a user's and the global or a country's preference profiles). Kullback-Leibler divergence is a well-established method to compare distributions (discrete preference profiles in our case); we employ rank correlation because conversion of feature values to ranks has already been proven successful for music similarity tasks [24].

We provide formulas for the specific measures in Table 3, where $\widehat{X}$ denotes the sum-to-unity normalized vector $X$ and $\operatorname{ranks}\left(P P_{U}^{W}\right)$ represents the real-valued preference profile converted to ranks, i.e. the vector containing all normalized item frequencies of user $u$, with respect to the frequency weighting approach $W(A F$ or $L F)$. When using AF.ILF, ranks $\left(P P_{u}^{W}\right)$ is extended to ranks $\left(P P_{u, c}^{A F \cdot I L F}\right)$, i.e. AF computed for user $u$, ILF on country $c$, or ranks $\left(P P_{c, g}^{A F I L F}\right)$, i.e. AF computed on country $c$, ILF globally. Note that we invert the results of the fraction-based formulations and the symmetrized KLdivergences in order to be consistent in that higher values always indicate closer to the mainstream, while lower ones indicate farther away from the mainstream.

\section{MUSIC RECOMMENDATION TAILORED TO USER MAINSTREAMINESS}

To evaluate the proposed mainstreaminess measures (cf. Section 3) with respect to their ability to improve performance in music recommendation, we conduct rating prediction experiments, which is a common approach to recommender systems evaluation. For this evaluation, we use the LFM- 1 b dataset of user-generated listening events from Last.fm [30], as detailed in the following.

\subsection{Data Preparation}

The LFM-1b dataset [30] covers 1,088,161,692 listening events of 120,322 unique users, who listened to $32,291,134$ unique tracks by $3,190,371$ unique artists. The core component of the dataset is the cleaned user-artist-playcount matrix (UAM) containing the number of listening events of 120,175 users to 585,095 unique artists. The distribution of listening events of the Last.fm data corresponds to a typical long-tail distribution [8].

As 65,132 user profiles do not contain any country information, we exclude those from our experiments since they do not contribute to defining a country's mainstreaminess. For each user in the remaining user set, we calculate the proposed mainstreaminess measures according to Table 3 . Note that using the LFM-1b dataset, the global population is in our case the Last.fm users in the dataset.

\subsection{Experimental Setup}

While we are aware that a truly user-centric evaluation would be beneficial for this kind of research, conducting a user study on tens of thousands of users (or even only a representative subset of the users) is beyond the scope of this paper. We therefore stick to the common approach of quantifying the performance of a recommender system by conducting a rating prediction task. To this end, we normalize and scale the playcount values in the UAM to the range $[0,1000]$ for each user individually, assuming that higher numbers of playcounts indicate higher user preference for an artist.

We apply the common singular value decomposition (SVD) method according to [27] to factorize the UAM and in turn effect rating prediction. In 5-fold cross-validation experiments, we use root mean square error (RMSE) and mean absolute error (MAE) as performance measures.

To obtain a baseline, we first run the rating prediction experiment on the global group of 65,132 users and report results of the error measures in the first row of Table 4. To study the influence of both, the different mainstreaminess definitions and mainstreaminess levels on recommendation performance, we then create subsets of users for each combination of mainstreaminess measure and country with at least 1,000 users. ${ }^{1}$ To this end, we split the users in each country into three (almost) equally sized subsets according to their mainstreaminess value: low corresponds to users in the lower 3-quantile (tertile) w.r.t. the respective mainstreaminess definition, mid and high, respectively, to the mid and upper tertile. In the individual experiments, all refers to the group of all users in each considered country, low only to the users in the lower 3-quantile (tertile) w.r.t. the respective mainstreaminess definition, mid and high defined analogously. Further, conducting the same experiment on all users in each country (user set all) allows for a comparison of a pure mainstreaminess filtering approach versus a combination of mainstreaminess filtering and demographic (country) filtering.

\subsection{Results and Discussion}

Table 4 shows the error measures (RMSE and MAE) for different definitions and levels of mainstreaminess, averaged over all considered countries (cf. Subsection 4.1), RMSE and MAE weighted by the number of users in the respective country. In the following

\footnotetext{
${ }^{1}$ The restriction to countries with at least 1,000 users was made to allow for a meaningful analysis, as performed in [31].
} 
discussion, we concentrate on RMSE since it is more common and considers larger differences between predicted and true ratings disproportionately more severe than smaller ones.

As a general finding, our results show that tailoring the recommendations to a user's mainstreaminess level (low, mid, high) leads to substantial error reductions, irrespective of the applied mainstreaminess measure. More specifically, $C_{c: A F, u: A F}$ outperforms the other measures in four regards: First, it leads to the lowest overall RMSE of 14.349 (all). Second, the errors realized by $C_{c: A F, u: A F}$ are also the lowest for each of the three user sets (low, mid, high). If better performance is achieved on a set with another measure, the difference is just in the third position after the decimal point. Third, $C_{c: A F, u: A F}$ performs on each of the three user sets (low, mid, high) in a balanced way (weighted RMSE amounts to respectively 3.692, 4.270, and 3.687), whereas the other mainstreaminess measures yield a rather unbalanced picture since each of them performs on at least one set far worse than on the other(s), e.g., $C_{g: A F, u: A F}$ with $19.183,7.443$, and 3.681, respectively, for low, mid, and high. Fourth, $C_{c: A F, u: A F}$ performs well also on the low mainstreaminess user set (low), which is a user segment that is typically difficult to satisfy.

The fraction-based approaches $F_{g: A F, u: A F}, F_{c: A F, u: A F}$, and $F_{g: A F, u: A F \cdot I L F}$ have in common that they perform far better in the high mainstreaminess segment than in the mid and the low one. This could indicate that these measures still privilege globally popular items too much and, thus, produce more errors in the mid and low segments.

Interestingly, the approaches based on symmetrized KullbackLeibler divergence $(D)$ perform worse when tailored towards a user's country $\left(D_{c: A F, u: A F}\right)$, compared to their application on a global level $\left(D_{g: A F, u: A F}\right)$. Combining the country-specific tailoring with the AF-ILF weighting allows for better results compared to applying both separately.

While our results do not suggest a general superiority of mainstreaminess measures that incorporate AF-ILF, first results of our deeper analysis on the country level indicate that these measures seem to perform particularly well for countries far away from the global mainstream, such as Finland (RMSE of $D_{c: A F \cdot I L F, u: A F \cdot I L F}$ for all=5.985, high=1.346, mid=1.365, low=1.418), but worse for high mainstream countries, such as the USA (RMSE of $D_{c: A F \cdot I L F, u: A F \cdot I L F}$ for all $=57.489$, high $=4.071$, mid $=4.077$, low $=55.968$ ). In the presented example, the low mainstream country Finland is small, and the respective weighted error measures in Table 4 do not reflect this country's users to the same extent as the large and high mainstream United States. As part of our ongoing large-scale analysis, delving into detail on country-specific aspects, we will investigate as a next step what factors influence the performance differences between countries for a given mainstreaminess measure.

A direct comparison of the RMSE achieved by our approach with the RMSE reported in [37], the work closest to ours, is unfortunately impossible since Vigliensoni and Fujinaga quantized playcounts into a 5-point Likert rating scale: $[1,5]$. Still, in a rough estimation, our results suggest that the accuracy of our best $C_{c: A F, u: A F}$ approach delivers a new benchmark in the combination of demographic (country) filtering and mainstreaminess filtering, with a RMSE of 14.3 on a [0,1000] scale. The best RMSE reported in [37] when considering mainstreamness and country information is approximately 0.9 on the much narrower [1,5] scale (cf. approach u.c.m. in Figure 2 of [37]).

\section{CONCLUSIONS AND OUTLOOK}

We proposed 11 novel measures to quantify the music mainstreaminess of a user, a country, and an entire population. Those are based on fractional $(F)$, divergence $(D)$, and rank correlation $(C)$ functions. Considering that music mainstream may be defined from a global but also a country-specific perspective, we particularly studied how the combination of a user's mainstreaminess and demographic (country) filtering influences the quality of music recommendations. Based on the LFM-1b dataset [30], we investigated the performance of the proposed measures in a rating prediction task, employing matrix factorization. To quantify performance, we computed countryaveraged, weighted RMSE and MAE figures for all mainstreaminess definitions and various mainstreaminess levels, and compared these with a global baseline. Overall, our results suggest that incorporating any kind of mainstreaminess information outperforms the baseline. Our best approach combines demographic filtering (based on a user profile's country) and mainstreaminess filtering based on Kendall's $\tau$ (variant $C_{c: A F, u: A F}$ ) and outperforms applying these filtering approaches separately. While our results do not hint at a general superiority of mainstreaminess measures that incorporate AF-ILF, they do show that such measures perform much better than others for countries whose preference profiles are far away from the global taste (e.g., Finland).

As part of future work, we will take an in-depth look at the differences between countries, i.e. analyze in which countries which mainstreaminess functions perform particularly well or poorly. Additionally, we plan to analyze how well our results generalize to other datasets providing demographic user information, e.g., the Spotify playlists dataset [23] or the Million Musical Tweets Dataset [15]. We further plan a user study to qualitatively investigate whether incorporating mainstreaminess information improves the perceived satisfaction with recommendations.

\section{ACKNOWLEDGMENTS}

This research is supported by the Austrian Science Fund (FWF): V579.

\section{REFERENCES}

[1] Hyung Jun Ahn. 2006. Utilizing Popularity Characteristics for Product Recommendation. International fournal of Electronic Commerce 11, 2 (2006), 59-80. http://www.jstor.org/stable/27751212

[2] Christian Anderson. 2006. The long tail: Why the future of business is selling less of more. Hachette Books, New York, NY, USA.

[3] Sarah Baker, Andy Bennett, and Jodie Taylor. 2013. Redefining mainstream popular music. Routledge.

[4] Chumki Basu, Haym Hirsh, and William Cohen. 1998. Recommendation as classification: Using social and content-based information in recommendation. In Proceedings of the AAAI Conference on Artificial Intelligence. American Association Intelligence, 714-720.

[5] Christine Bauer, Marta Kholodylo, and Christine Strauss. 2017. Music Recommender Systems: Challenges and Opportunities for Non-Superstar Artists. In Proceedings of 30th Bled eConference, Andreja Pucihar, Mirjana Kljajić Borštnar, Christian Kittl, Pascal Ravesteijn, Roger Clarke, and Roger Bons (Eds.). University of Maribor Press, Bled, Slovenia, 21-32.

[6] Erik Brynjolfsson, Yu Hu, and Duncan Simester. 2011. Goodbye Pareto Principle, Hello Long Tail: The Effect of Search Costs on the Concentration of Product 
Sales. Management Science 57, 8 (2011), 1373-1386. http://www.jstor.org/stable/ 25835786

[7] Oliver Budzinski and Julia Pannicke. 2017. Do preferences for pop music converge across countries?:Empirical evidence from the Eurovision Song Contest. Creative Industries fournal (2017), 1-20.

[8] Ôscar Celma. 2010. Music Recommendation and Discovery. Springer, Berlin, Heidelberg, Germany.

[9] Òscar Celma and Pedro Cano. 2008. From Hits to Niches?: Or How Popular Artists Can Bias Music Recommendation and Discovery. In Proceedings of the v2Nd KDD Workshop on Large-Scale Recommender Systems and the Netflix Prize Competition (NETFLIX 2008). ACM, New York, NY, USA, Article 5, 8 pages. https: //doi.org/10.1145/1722149.1722154

[10] Zhiyong Cheng and Jialie Shen. 2014. Just-for-Me: An Adaptive Personalization System for Location-Aware Social Music Recommendation. In Proceedings of the International Conference on Multimedia Retrieval (ICMR 2014). ACM, New York, NY, USA, 185:185-185:192. https://doi.org/10.1145/2578726.2578751

[11] Charles L.A. Clarke, Maheedhar Kolla, Gordon V. Cormack, Olga Vechtomova, Azin Ashkan, Stefan Büttcher, and Ian MacKinnon. 2008. Novelty and Diversity in Information Retrieval Evaluation. In Proceedings of the 31st Annual International ACM SIGIR Conference on Research and Development in Information Retrieval (SIGIR 2008). ACM, New York, NY, USA, 659-666. https://doi.org/10.1145/1390334. 1390446

[12] Paolo Cremonesi, Franca Garzotto, Roberto Pagano, and Massimo Quadrana 2014. Recommending without short head. In Proceedings of the 23rd International Conference on World Wide Web (WWW 2014). ACM, 245-246.

[13] Katayoun Farrahi, Markus Schedl, Andreu Vall, David Hauger, and Marko Tkalčič 2014. Impact of Listening Behavior on Music Recommendation. In Proceedings of the 15th International Society for Music Information Retrieval Conference (ISMIR 2014). 483-488.

[14] Bruce Ferwerda. 2016. Improving the User Experience of Music Recommender Systems Through Personality and Cultural Information. PhD. Johannes Kepler University Linz, Linz, Austria.

[15] David Hauger, Markus Schedl, Andrej Košir, and Marko Tkalčič. 2013. The Million Musical Tweets Dataset: What Can We Learn From Microblogs. In Proceedings of the 14th International Society for Music Information Retrieval Conference (ISMIR 2013).

[16] Xiao Hu, Jin Ha Lee, Kahyun Choi, and J.S. Downie. 2014. A Cross-cultural Study of Mood in K-pop Songs. In Proceedings of the 15th International Society for Music Information Retrieval Conference (ISMIR 2014). 217-238.

[17] Shinobu Kitayama and Hyekyung Park. 2007. Cultural shaping of self, emotion, and well-being: How does it work? Social and Personality Psychology Compass 1, 1 (2007), 202-222.

[18] Rajeev Kumar, B.K. Verma, and Shyam Sunder Rastogi. 2014. Social popularity based SVD++ recommender system. International fournal of Computer Applications 87, 14 (2014), 33-37.

[19] Audrey Laplante. 2014. Improving music recommender systems: what can we learn from research on music tags?. In Proceedings of the 15th International Society for Music Information Retrieval Conference (ISMIR 2014). 451-456.

[20] Greg Linden, Brent Smith, and Jeremy York. 2003. Amazon.com Recommendations: Item-to-Item Collaborative Filtering. IEEE Internet Computing 4, 1 (2003), $76-80$.

[21] Brian McFee, Luke Barrington, and Gert Lanckriet. 2012. Learning content similarity for music recommendation. IEEE transactions on audio, speech, and language processing 20, 8 (2012), 2207-2218.

[22] Miquel Montaner, Beatriz López, and Josep Lluís de la Rosa. 2003. A taxonomy of recommender agents on the Internet. Artificial Intelligence Review 19, 4 (2003), 285-330.

[23] Martin Pichl, Eva Zangerle, and Günther Specht. 2015. Towards a Context-Aware Music Recommendation Approach: What is Hidden in the Playlist Name? In Proceedings of the 15th IEEE International Conference on Data Mining Workshop (ICDM 15). IEEE, Piscataway, NJ, USA, 1360-1365.

[24] Tim Pohle, Markus Schedl, Peter Knees, and Gerhard Widmer. 2006. Automatically Adapting the Structure of Audio Similarity Spaces. In Proceedings of the 1st Workshop on Learning the Semantics of Audio Signals (LSAS 2006).

[25] Francesco Ricci, Lior Rokach, and Bracha Shapira. 2015. Recommender Systems Handbook (2nd ed.). Springer, New York, NY.

[26] Paul Rutten. 1991. Local popular music on the national and international markets Cultural Studies 5, 3 (1991), 294-305.

[27] Ruslan Salakhutdinov and Andriy Mnih. 2007. Probabilistic Matrix Factorization. In Proceedings of the 20th International Conference on Neural Information Processing Systems (NIPS 2007). Curran Associates Inc., 1257-1264. http://dl.acm.org/citation. cfm?id=2981562.2981720

[28] Gerard Salton, Andrew Wong, and Chung-Shu Yang. 1975. A Vector Space Model for Automatic Indexing. Commun. ACM 18, 11 (1975), 613-620. https: //doi.org/10.1145/361219.361220

[29] Markus Schedl. 2013. Ameliorating Music Recommendation: Integrating Music Content, Music Context, and User Context for Improved Music Retrieval and
Recommendation. In Proceedings of International Conference on Advances in Mobile Computing \& Multimedia (MoMM) (MoMM '13). ACM, New York, NY, USA, 3:3-3:9. https://doi.org/10.1145/2536853.2536856

[30] Markus Schedl. 2016. The LFM-1b Dataset for Music Retrieval and Recommendation. In Proceedings of the 17th ACM International Conference on Multimedia Retrieval (ICMR 2016). New York, NY, USA.

[31] Markus Schedl. 2017. Investigating country-specific music preferences and music recommendation algorithms with the LFM-1b dataset. International fournal of Multimedia Information Retrieval 6, 1 (2017), 71-84. https://doi.org/10.1007/ s13735-017-0118-y

[32] Markus Schedl and Christine Bauer. 2017. Distance- and Rank-based Music Mainstreaminess Measurement. In Adjunct Publication of the 25th Conference on User Modeling, Adaptation and Personalization (July 9-12, 2017) (UMAP 2017). ACM, New York, NY, USA, 364-367. https://doi.org/10.1145/3099023.3099098

[33] Markus Schedl, Emilia Gómez, and Julián Urbano. 2014. Music Information Retrieval: Recent Developments and Applications. Foundations and Trends in Information Retrieval 8, 2-3 (2014), 127-261. https://doi.org/10.1561/1500000042

[34] Markus Schedl and David Hauger. 2015. Tailoring Music Recommendations to Users by Considering Diversity, Mainstreaminess, and Novelty. In Proceedings of the 38th Annual International ACM SIGIR Conference on Research and Development in Information Retrieval (SIGIR 2015).

[35] Markus Schedl, Peter Knees, Brian McFee, Dmitry Bogdanov, and Marius Kaminskas. 2015. Recommender Systems Handbook (2nd ed.). Springer, New York, NY, USA, Chapter Music Recommender Systems, 453-492.

[36] Catherine J. Stevens. 2012. Music Perception and Cognition: A Review of Recent Cross-Cultural Research. Topics in Cognitive Science 4, 4 (2012), 653-667.

[37] Gabriel Vigliensoni and Ichiro Fujinaga. 2016. Automatic music recommendation systems: do demographic, profiling, and contextual features improve their performance?. In Proceedings of the 17th International Society for Music Information Retrieval Conference (August 7-11, 2016) (ISMIR 2016). 94-100.

[38] Linxing Xiao, Lie Lu, Frank Seide, and Jie Zhou. 2009. Learning a music similarity measure on automatic annotations with application to playlist generation. In Proceedings of the IEEE International Conference on Acoustics, Speech and Signal Processing (April 19-24, 2009) (ICASSP 2009). IEEE, 1885-1888.

[39] Yan Yan, Tianlong Liu, and Zhenyu Wang. 2015. A Music Recommendation Algorithm Based on Hybrid Collaborative Filtering Technique. In 4th National Conference on Social Media Processing (November 16-17, 2015) (SMP 2015), Xichun Zhang, Maosong Sun, Zhenyu Wang, and Xuanjing Huang (Eds.). Springer, Singapore, 233-240. https://doi.org/10.1007/978-981-10-0080-5_23

[40] JungAe Yang. 2016. Effects of Popularity-Based News Recommendations ("MostViewed") on Users' Exposure to Online News. Media Psychology 19, 2 (2016), 243-271. https://doi.org/10.1080/15213269.2015.1006333

[41] Yuan Cao Zhang, Diarmuid Ó. Seaghdha, Daniele Quercia, and Tamas Jambor. 2012. Auralist: Introducing Serendipity into Music Recommendation. In Proceedings of the 5th ACM International Conference on Web Search and Data Mining (WSDM 2012). Seattle, WA, USA. 


\begin{tabular}{|c|c|c|c|}
\hline Mainstreaminess & user set & w.RMSE & w.MAE \\
\hline Baseline (global UAM) & & 29.105 & 25.202 \\
\hline \multirow[t]{4}{*}{$F_{g: A F, u: A F}$} & all & 26.377 & 24.050 \\
\hline & high & 3.714 & 1.308 \\
\hline & mid & 12.574 & 9.887 \\
\hline & low & 14.186 & 11.625 \\
\hline \multirow[t]{4}{*}{$F_{g: A F, u: A F \cdot I L F}$} & all & 21.137 & 18.617 \\
\hline & high & 3.681 & 1.299 \\
\hline & mid & 11.035 & 8.191 \\
\hline & low & 14.426 & 11.868 \\
\hline \multirow{4}{*}{$F_{g: A F \cdot I L F, u: A F \cdot I L F}$} & all & 19.140 & 16.769 \\
\hline & high & 11.777 & 9.121 \\
\hline & mid & 13.396 & 10.833 \\
\hline & low & 8.708 & 5.806 \\
\hline \multirow[t]{4}{*}{$F_{c: A F, u: A F}$} & all & 14.465 & 11.958 \\
\hline & high & 3.723 & 1.309 \\
\hline & mid & 8.681 & 6.112 \\
\hline & low & 12.706 & 9.952 \\
\hline \multirow[t]{4}{*}{$F_{c: A F \cdot I L F, u: A F \cdot I L F}$} & all & 17.615 & 15.301 \\
\hline & high & 9.237 & 6.648 \\
\hline & mid & 3.686 & 1.305 \\
\hline & low & 10.122 & 7.610 \\
\hline \multirow[t]{4}{*}{$D_{g: A F, u: A F}$} & all & 24.026 & 21.705 \\
\hline & high & 10.561 & 8.024 \\
\hline & mid & 9.854 & 7.299 \\
\hline & low & 5.365 & 2.909 \\
\hline \multirow[t]{4}{*}{$D_{c: A F, u: A F}$} & all & 28.021 & 25.746 \\
\hline & high & 5.365 & 2.912 \\
\hline & mid & 13.510 & 10.840 \\
\hline & low & 25.923 & 22.621 \\
\hline \multirow[t]{4}{*}{$D_{c: A F \cdot I L F, u: A F \cdot I L F}$} & all & 14.628 & 11.624 \\
\hline & high & 3.656 & 1.281 \\
\hline & mid & 7.035 & 4.515 \\
\hline & low & 8.589 & 5.670 \\
\hline \multirow[t]{4}{*}{$C_{g: A F, u: A F}$} & all & 15.906 & 13.525 \\
\hline & high & 3.680 & 1.291 \\
\hline & mid & 7.443 & 4.472 \\
\hline & low & 19.183 & 16.373 \\
\hline \multirow[t]{4}{*}{$C_{c: A F, u: A F}$} & all & 14.349 & 12.032 \\
\hline & high & 3.687 & 1.290 \\
\hline & mid & 4.270 & 1.833 \\
\hline & low & 3.692 & 1.308 \\
\hline \multirow[t]{4}{*}{$C_{c: A F \cdot I L F, u: A F \cdot I L F}$} & & 30.827 & 28.535 \\
\hline & high & 7.680 & 5.187 \\
\hline & mid & 4.825 & 2.340 \\
\hline & low & 10.785 & 8.1084 \\
\hline
\end{tabular}

Table 4: Weighted root mean square error (RMSE) and weighted mean absolute error (MAE) for various mainstreaminess definitions and levels, i.e. user sets. Rating values are scaled to $[0,1000]$. Experiments are conducted on the country level (except for first row using the complete UAM irrespective of country) and error measures are averaged (arithmetic mean) over all countries with more than 1,000 users and weighted by number of users in the respective country. In the individual experiments, all refers to the group of all users in each considered country, low only to the users in the lower 3-quantile (tertile) w.r.t. the respective mainstreaminess definition, mid and high defined analogously.

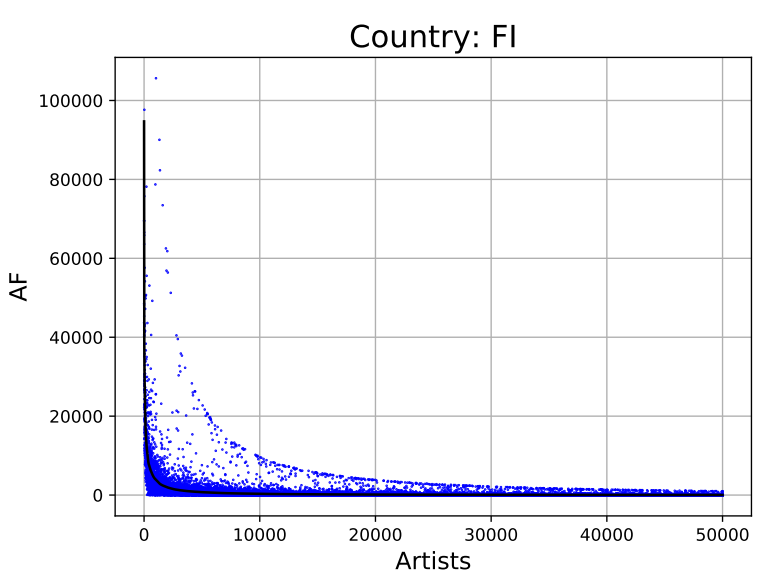

(a) Artist frequency (AF)

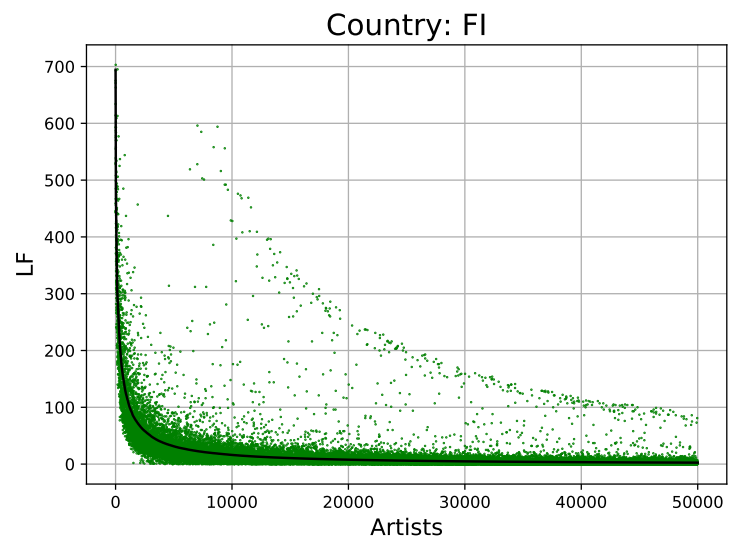

(b) Listener frequency (LF)

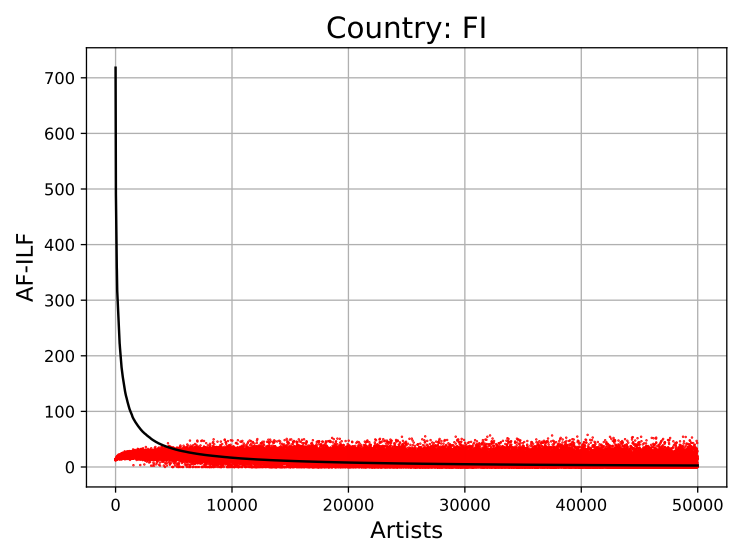

(c) Artist frequency-inverse listener frequency (AF-ILF)

Figure 1: Artist frequency (AF), listener frequency (LF), and artist frequency-inverse listener frequency (AF-ILF) for the top 50,000 artists in Finland. Artist IDs (x-axis) are sorted by global AF, LF, or AF-ILF values, respectively. The black line indicates the global values. 